\title{
Valoración Psicométrica del Cuestionario de Acoso Psicológico Percibido
}

\section{Psychometric Evaluation of the Perceived Mobbing Questionnaire}

\author{
Consuelo Morán \\ Universidad de León
}

\author{
Mónica Teresa González \\ Universidad Autónoma de Nuevo León
}

\author{
René Landero \\ Universidad Autónoma de Nuevo León
}

\begin{abstract}
Resumen. El propósito de este estudio fue desarrollar y examinar algunas de las propiedades psicométricas del Cuestionario de Acoso Psicológico Percibido (CAPP). El CAPP es una medida de autoinforme de 15 ítems, con formato de respuesta del tipo Likert en una escala de cinco puntos, diseñado para evaluar el sentimiento de acoso psicológico en el lugar de trabajo. Una muestra de 390 trabajadores en servicios humanos respondieron al CAPP juntamente con otras escalas de salud y burnout. Los datos fueron analizados con técnicas apropiadas de análisis de ítems y factorización. Los resultados mostraron una satisfactoria confiabilidad del CAPP $(\alpha=.92)$, así como una clara estructura factorial unidimensional de la escala. Por fin, las correlaciones entre mobbing percibido, burnout y salud pueden servir de evidencia de validez de la escala.

Palabras clave: acoso psicológico, validez, fiabilidad, análisis factorial, salud

Abstract. The aim of this study was to develop and to examine some psychometric properties of the Perceived Mobbing Questionnaire, a 15-item self-report questionnaire designed to measure the feeling of workplace mobbing. A sample of 390 workers in human services completed the Perceived Mobbing Questionnaire and additional self-report assessments of health and burnout. The data were analysed both by items and by scales, using more appropriate factoring techniques. Results showed a high internal consistency for CAPP reliability $(\alpha=.92)$, as well as a clear one-factor structure of the scale. Lastly, the correlations of mobbing with burnout and health can be seen as evidence of validity.

Key words: mobbing, validity, reliability, factor analysis, health.
\end{abstract}

El estudio del acoso psicológico en el trabajo en España es reciente $\mathrm{y}$, aunque el interés por el tema ha crecido sustancialmente en los últimos años, probablemente, como señalan Moreno-Jiménez y RodríguezMuñoz (2006) los estudios científicos sean aún insuficientes. La mayoría de los trabajos que se venían publicando eran de carácter divulgativo, pero sirvieron para plantear esa importante cuestión, tanto a nivel de concienciación social, así como en el campo de la jurisprudencia y en el de la prevención de riesgos laborales. Las definiciones de acoso psicológico en el trabajo (Salin, 2003) consideran al acoso como una forma de agresión interpersonal, o una conducta hostil y antisocial en el ambiente de trabajo y, en este sentido, se han utilizado varios términos para describir este tipo de agresión interpersonal. Por ejemplo, entre los investigadores del Reino Unido e Irlanda, así como también entre los de Australia y el norte de Europa, predomina el uso del término bullying (ver Einarsen, Hoel, Zapf y Cooper, 2003), siendo los alemanes quienes han utilizado el término mobbing para el mismo fenómeno (LaVan y Martin, 2007). En Turquía, Unal y Karaadhmet, (2007) usan la etiqueta mobbing en el cuestionario NAQRutilizado para medir este fenómeno y sus cinco cate-

La correspondencia sobre este artículo debe enviarse a la primera autora a la Universidad de León, Facultad de Ciencias del Trabajo, Campus de Vagazana, s/n 24071, León. E-mail: consuelo.moran@unileon.es gorías de conductas. Otros autores, como Zapf (1999), usan ambos términos de un modo intercambiable, como se hace también en el número monográfico "Acoso Psicológico en el Trabajo", que se presenta en inglés como Special issue on mobbing in the workplace (Moreno-Jiménez y Rodríguez-Muñoz, 2006). En esta publicación unos artículos la denominación workplace mobbing en su título en inglés (v.g., Einarsen y Hauge, 2006) y otros workplace bullying (v.g., MorenoJiménez, Rodríguez-Muñoz, Moreno y Garrosa, 2006). En los Estados Unidos se emplean también otros términos además del workplace bullying, como son, por ejemplo, emotional abuse (Keashly, 1998) y workplace aggression (Neuman y Baron, 1998).

Entre quienes prefieren el uso de workplace bullying, hace también equiparable esta expresión a harassment (v.g., Salin, 2008), y en ambos casos como una forma de interacción negativa que puede expresarse de muchas maneras, desde la agresión verbal y excesiva crítica o monitorizar el trabajo, al aislamiento social o al trato silencioso. De este modo, dice Salin (2008), el bullying/harassment es así una cuestión de acumulación de muchos actos menores, que se convierten en un patrón de maltrato sistemático. En Europa, Hanz Leymann $(1990,1996)$ fue pionero en el estudio del maltrato en el ámbito laboral y lo denominó con el término mobbing. Según este autor, el concepto de mobbing define el encadenamiento, durante 
un largo periodo de tiempo, de palabras malintencionadas y artimañas hostiles, expresadas y manifestadas por una o varias personas hacia una tercera (la víctima) poniéndola en una situación de desventaja con alto riesgo de ser despedida de su trabajo (Leymann, 1996). Para que pueda denominarse mobbing se tienen que dar, según este autor, tres condiciones: 1) los actos han de ser sentidos como hostiles, 2) se repiten frecuentemente (al menos una vez por semana) y 3 ) se prolongan durante un cierto periodo de tiempo (por lo menos seis o más meses). De acuerdo con Einarsen et al. (2003), en el contexto laboral, mobbing significa irritar, ofender, excluir socialmente a alguien o dificultarle la realización de su trabajo. Actos aislados que resultan molestos, como el asignar tareas por debajo de las propias competencias, ser objeto de bromas por parte de compañeros o que no te avisen para ir a tomar café con ellos, pueden ser considerados como episodios cotidianos en el entorno de trabajo, pero no mobbing Cuando se repiten de manera sistemática durante un tiempo prolongado, dando lugar a un ambiente de trabajo desagradable y hostil para quien lo padece, pueden considerarse como comportamientos de acoso (Salin, 2006). A menudo las víctimas encuentran dificultades para defenderse, ya que se hallan en ese momento de su vida en una situación real o percibida de inferioridad. La situación de inferioridad puede deberse a múltiples causas, como ser de menor envergadura física, tener una posición aún no consolidada en la empresa o tener pocos compañeros que apoyan, según un estudio realizado por Bowling y Beehr (2006). Además, las víctimas de mobbing pueden estar expuestas a una amplia gama de conductas de tipo directo o indirecto, en forma de insultos persistentes o comentarios ofensivos, críticas constantes, abusos personales o físicos reiterados. A otros se les da un tratamiento silencioso, experimentando así aislamiento y exclusión social. El común denominador de estas conductas es que se utilizan con el propósito de humillar, intimidar, aterrar o castigar a la víctima. En la mayoría de las víctimas de mobbing es central el sentimiento de ser tratadas injustamente (Mikkelsen y Einarsen, 2002).

Otro reto importante que se añade a la definición de acoso en el trabajo, es el de su medición. La frecuencia exacta del acoso varía mucho de unos estudios a otros (Zapf, Einarsen, Hoel y Vartia, 2003), ya que utilizan diferentes criterios cuando deciden quién es una víctima y quién no. Actualmente existen dos aproximaciones a la hora de medir el acoso psicológico en el trabajo (Einarsen et al., 2003): (1) la medición subjetiva que evalúa la percepción de ser víctima de acoso; y (2) la medición objetiva o exposición percibida a conductas especificas de acoso. La primera aproximación se basa en un método de auto-etiqueta, según el cual se pide a los participantes que indiquen si ellos se perciben a sí mismos como víctimas de mobbing o no. La mayor parte de las veces se les presenta con una definición del término. Esta aproximación es conocida en la literatura del mobbing como método subjetivo, o de autoevaluación, ya que se basa en la percepción subjetiva de quien responde al cuestionario. La otra aproximación deriva de los trabajos de Leymann (1990), la cual presenta a los participantes una serie de ítems que miden la exposición a determinadas conductas especificadas de acoso. Este método, denominado operacional, objetivo o basado en criterio (Notelaers, Einarsen, De Witte y Vermunt, 2006), considera víctimas de acoso a quienes responden que están siendo objeto de al menos un acto especificado en el cuestionario, semanal o diariamente, desde hace por lo menos 6 meses.

Los instrumentos de medida más comúnmente utilizados según este criterio son el Leymann Inventory of Psychological Terror-LIPT (Leymann, 1990), adaptado al castellano por González de Rivera y Rodríguez (2003) y el Negative Acts Questionnaire (NAQ; Einarsen y Hoel, 2001). En España, Moreno-Jiménez, Rodríguez-Muñoz, Garrosa y Morante (2005) han empleado un cuestionario mixto, dividiendo las conductas típicas de acoso en categorías (aislamiento social, desacreditación, exigencia extrema, consecuencias) y pidiendo al sujeto que conteste el grado en que padece esas conductas de acoso en una escala de 0 (Nunca) a 4 (Continuamente). Ambos métodos han sido objeto de numerosas críticas (ver Hoel, Rayner y Cooper, 1999; Mikkelsen y Einarsen, 2001; Salin, 2006). Por ejemplo, cuando se utiliza el método objetivo y el de auto-etiqueta combinados, ni siquiera la mitad de los participantes categorizados como víctimas se consideran (según el método subjetivo) a sí mismas como tales. Cuando se utiliza el método objetivo en los estudios, la frecuencia de acoso varía entre un $8 \%$ y un $15 \%$, mientras que cuando se utilizan métodos subjetivos para estimar la proporción de las víctimas la frecuencia está entre un 2 y un 10\% (Zapf et al., 2003). Además, existen otras razones para cuestionar la validez del método objetivo. En primer lugar, el punto de corte proporcionado por este método para considerar que alguien padece o no mobbing se escoge de manera arbitraria. En segundo lugar, cada método utiliza un número de actos diferentes (desde 26 en el NAQ a 45 en el LIPT), lo que puede determinar que el número de víctimas halladas dependa realmente del listado de actos negativos que se hayan utilizado para realizar el cuestionario. Por otra parte, no todos los actos revisten el mismo nivel de gravedad, de tal manera que en ocasiones incluso un bajo nivel de exposición a determinados actos negativos puede considerarse acoso en el trabajo (Notelaers et al., 2006).

La medida que hemos creado con el fin de evaluar el mobbing, como es el Cuestionario de Acoso Psicológico Percibido (CAPP), se enmarca en la primera aproximación, es decir, en la del método subjetivo. Consideramos que, de las tres condiciones que propone Leymann (1996) para que pueda denominarse mobbing, la primera "los actos negativos han de ser sentidos como hostiles" es fundamental a nivel psico- 
lógico. A diferencia de las escalas e inventarios anteriores, que suelen ser de ítem único, nuestra escala está compuesta de 15 ítems, de los que sólo uno pregunta directamente si el informante cree que está siendo acosado en el lugar de trabajo. Los demás aluden a sentirse inseguros y amenazados, percibir agresividad, sentirse mal entre los compañeros, percibir un clima laboral de intimidación, humillación y propenso al acoso. También el CAPP se diferencia de anteriores instrumentos de medida del acoso en el trabajo por el tipo de respuesta. Hemos tenido en cuenta la propuesta de Matthiesen, Raknes y Røkkum (1989), quienes consideran que el mobbing debe ser visto como un continuo que va desde "nada expuesto" hasta "muy expuesto", no como un "sî" o "no" ocurre este fenómeno. No siempre es fácil hallar el punto de corte que clasifique a las víctimas de las no víctimas, como tampoco para ellas mismas es fácil ponerse la etiqueta de acosadas o la de no acosadas. En su reciente revisión de la literatura, Einarsen y Hauge (2006) insisten en que la definición operativa para diferenciar entre casos severos de acoso laboral y la exposición al mismo de forma menos intensa (la que puede considerarse una forma de estrés social en el trabajo) no posee un claro criterio de corte. A los 15 ítems del Cuestionario de Acoso Psicológico Percibido (CAPP) se responde con puntuaciones en un continuo, desde "no lo siento en absoluto" hasta "siempre lo siento", pasando por tres grados intermedios.

Con el fin de estudiar si son adecuadas sus propiedades psicométricas, nos hemos propuesto en el presente trabajo los siguientes objetivos: (1) la fiabilidad del Cuestionario de Acoso Psicológico Percibido, (2) evaluar la estructura factorial del cuestionario, y (3) examinar las correlaciones entre el CAPP, el burnout y la salud en una muestra de trabajadores españoles. Las correlaciones se muestran como evidencias de validez del CAPP al compararlo con los constructos también medidos en este estudio, especialmente con las medidas de distrés psicológico del GHQ-28, ya empleado este cuestionario en otros estudios de mobbing (v.g. Moreno-Jiménez et al., 2006; García-Izquierdo, Llor, García Izquierdo y Ruiz, 2006).

\section{Método}

\section{Participantes}

Respondieron a los cuestionarios 390 trabajadores de servicios humanos: la muestra incluía a profesores (74\%) y cuidadores, enfermeros, médicos, jefes de personal y vendedores (26\%), todos ellos de la provincia de León (España). El 35\% fueron varones. La media de edad fue de 38 años (rango de 21 a 65). Llevaban trabajando una media de 13 años (rango desde 1 a 40 años). Por nivel de educación, el $8 \%$ tenían estudios básicos, el $15 \%$ de bachiller y el $77 \%$ estudios universitarios.

\section{Procedimiento}

Se utilizó un muestreo aleatorio-accidental (Kerlinger, 2001). Los cuestionarios fueron aplicados por personas expertas (psicólogos y psicopedagogos en ejercicio, estudiantes de doctorado en el programa "Psicología y Ciencias de la Educación") a compañeros de trabajo y personas allegadas. Respondieron a los cuestionarios durante su tiempo libre, en casa. Se realizaron análisis descriptivos para todas las variables; análisis de consistencia interna, análisis factorial exploratorio para el Cuestionario de Acoso Psicológico Percibido. Para el análisis factorial exploratorio (AFE) (con SPSS), el método de extracción empleado fue el de componentes principales (PCA) con rotación ortogonal (varimax), para una primera aproximación a la estructura factorial de la escala. Para evaluar las correlaciones entre las variables se utilizó la correlación de Spearman, debido a que los datos no se distribuyeron con normalidad (ver Tabla 1).

\section{Medidas}

Cuestionario de Acoso Psicológico Percibido (CAPP) (Morán, 2007). Evalúa acoso psicológico en el trabajo. Consta de 15 ítems que se responden en una escala Likert de cinco puntos, donde 1 significa "no lo siento en absoluto" y 5 "siempre lo siento", con puntuaciones intermedias. Los ítems fueron elaborados y sometidos a juicio de expertos y, de 20 ítems iniciales, se mantuvieron 15, evitando la reiteración de los contenidos y que fuera excesivamente largo. El ítem 13 alude directamente a sentirse acosado, siendo su redacción textual: "Siento como si me estuvieran acosando en mi lugar de trabajo". El coeficiente de fiabilidad del CAPP en este estudio fue de $\alpha=.92$. A mayor puntuación en el CAPP, mayor sentimiento de acoso en el lugar de trabajo.

Inventario de Burnout de Maslach (MBI). Para la evaluación del burnout se utilizó el Maslach Burnout Inventory (MBI-HSS), versión para profesionales de servicios humanos (Maslach y Jackson, 1986). Este inventario consta de 22 ítems que evalúan las tres dimensiones del burnout: Cansancio emocional (9 ítems), Despersonalización (5 ítems) y Realización personal (8 ítems). Los ítems se contestan señalando desde 0 (nunca) hasta 6 (me ocurre todos los días), en una escala de 7 grados en la que indican la frecuencia con la que han experimentado la situación descrita durante el último año. Los valores de fiabilidad de las escalas en nuestro estudio fueron $\alpha=.79$ para realización personal en el trabajo, $\alpha=.83$ para agotamiento emocional y $\alpha=.54$ para despersonalización. Las puntuaciones altas en cansancio emocional y despersonalización y bajas en realización personal indican más burnout.

Cuestionario de Salud General de Goldberg (GHQ28) (Goldberg y Williams, 1996). Este cuestionario 
ofrece una medida del distrés psicológico. Se compone de 28 ítems y se ha diseñado como una medida de salud y psicopatología a través de cuatro escalas de 7 ítems cada una: síntomas somáticos, ansiedad e insomnio, disfunción social y depresión severa. Estas escalas pueden utilizarse por separado, o bien pueden servir para la obtención de una puntuación global del GHQ28 a través de la última escala denominada salud general, que es el resultado de sumar las puntuaciones totales de las otras cuatro escalas. Goldberg y Williams (1996) permiten varios modos de puntuación de los ítems, siendo muy utilizado el que usamos en este estudio: una escala de tipo Likert de 0 a 3, indicando el 0 "No, en absoluto" y el 3 "Mucho más (o mejor) de lo habitual". Cuánto más alta sea la puntuación obtenida en las escalas, desde 0 a 21 puntos en cada una, más falta de salud está experimentando el individuo. La puntuación total del GHQ-28 podría alcanzar los 84 puntos. Los valores de fiabilidad de las escalas en nuestro estudio fueron $\alpha=.82$ para síntomas somáti$\cos , \alpha=.90$ para ansiedad e insomnio y $\alpha=.76$ para disfunción social, $\alpha=.85$ para depresión severa, $\mathrm{y}$ $\alpha=.92$ para toda la prueba.

\section{Resultados}

En la Tabla 1 se muestran las medidas de tendencia central y la prueba de Kolmogorov-Smirnov que evidencia la falta de normalidad en las variables. Considerando las medidas de tendencia central y los puntajes máximos posibles de las escalas, puede observarse que los sujetos reportan puntuaciones alejadas del máximo posible (75 puntos) para acoso psicológico (mobbing), y lo mismo sucede para las subescalas del GHQ: síntomas somáticos, ansiedad e insomnio, disfunción social y depresión se encuentran lejos del máximo puntaje posible (21 puntos); asimismo, el puntaje de salud general que hace referencia al distrés psicológico se aleja de la puntuación máxima posible: 84 puntos. En cuanto a la escala de burnout, los puntajes promedio de cansancio emocional y despersonalización se alejan de los máximos posibles (54 y 30 respectivamente); por el contrario, el puntaje promedio (38) en el área de realización personal se acerca al máximo posible de 48.

\section{Análisis factorial exploratorio}

En el análisis factorial exploratorio realizado para el Cuestionario de Acoso Psicológico Percibido, el valor de KMO (.937) es alto y adecuado, la prueba de esfericidad fue significativa $\left(\chi^{2}=2883.86 ; \mathrm{gl}=105 ; \mathrm{p}=\right.$ $.001)$. El número sugerido de factores por el criterio de autovalor mayor a 1 es de 2 factores, sin embargo, el gráfico de sedimentación (figura 1) sugiere un factor, el cual explica el $47.78 \%$ de la varianza, el segundo factor aportaría $7.52 \%$ de varianza. El alfa de Cronbach de esta escala fue de .92 .

La saturación de cada ítem en el AFE para ambas soluciones (unifactorial y bifactorial) se presenta en la Tabla 2. Solo 2 ítems saturan adecuadamente en el segundo factor, el ítem 1 claramente pertenece al segundo factor, el ítem 3 satura en ambos factores. Así, se considera que la mejor solución es la unifactorial. Observando la Tabla 2, vemos que la idea de la posibilidad de hacerle a uno la vida imposible (según los ítems 11 y 6), obtiene el mayor peso factorial del CAPP. Con menor peso se halla la tensión en el ambiente por discusiones entre compañeros (ítem 2). En posición intermedia se halla el ítem 13, que alude expresamente al sentimiento de acoso en el lugar de trabajo.

\section{Validez}

Para contar con datos de validez, se evaluaron las correlaciones con las 3 subescalas de burnout y con las subescalas deL GHQ-28. Tal como se esperaba, sólo se encontró correlación negativa con la variable de reali-

Tabla 1: Estadísticos descriptivos y prueba de normalidad

\begin{tabular}{lcrrr}
\hline Variable & Mediana & Media & DT & K-S \\
\hline Mobbing (CAPP) & 26.0 & 27.40 & 9.67 & $.137 ; \mathrm{p}=.001$ \\
Salud general (distrés psicológico) & 15.0 & 18.63 & 10.27 & $.156 ; \mathrm{p}=.001$ \\
Síntomas somáticos & 4.5 & 5.47 & 14.51 & $.162 ; \mathrm{p}=.009$ \\
Ansiedad e insomnio & 4.0 & 5.16 & 4.19 & $.135: \mathrm{p}=.001$ \\
Disfunción social & 7.0 & 6.81 & 2.22 & $.261 ; \mathrm{p}=.001$ \\
Depresión & 0 & 1.24 & 2.26 & $.309 ; \mathrm{p}=.001$ \\
Burnout & & & 10.0 & $.051 ; \mathrm{p}=.001$ \\
Cansancio emocional & 19.0 & 19.83 & 4.88 & $.121 ; \mathrm{p}=.001$ \\
Despersonalización & 5.0 & 5.71 & 7.61 & $.099 ; \mathrm{p}=.001$ \\
Realización personal & 38.0 & 36.40 & & \\
\hline
\end{tabular}


Figura 1. Gráfico de sedimentación para el Cuestionario de Acoso Psicológico Percibido

\section{Gráfico de sedimentación}

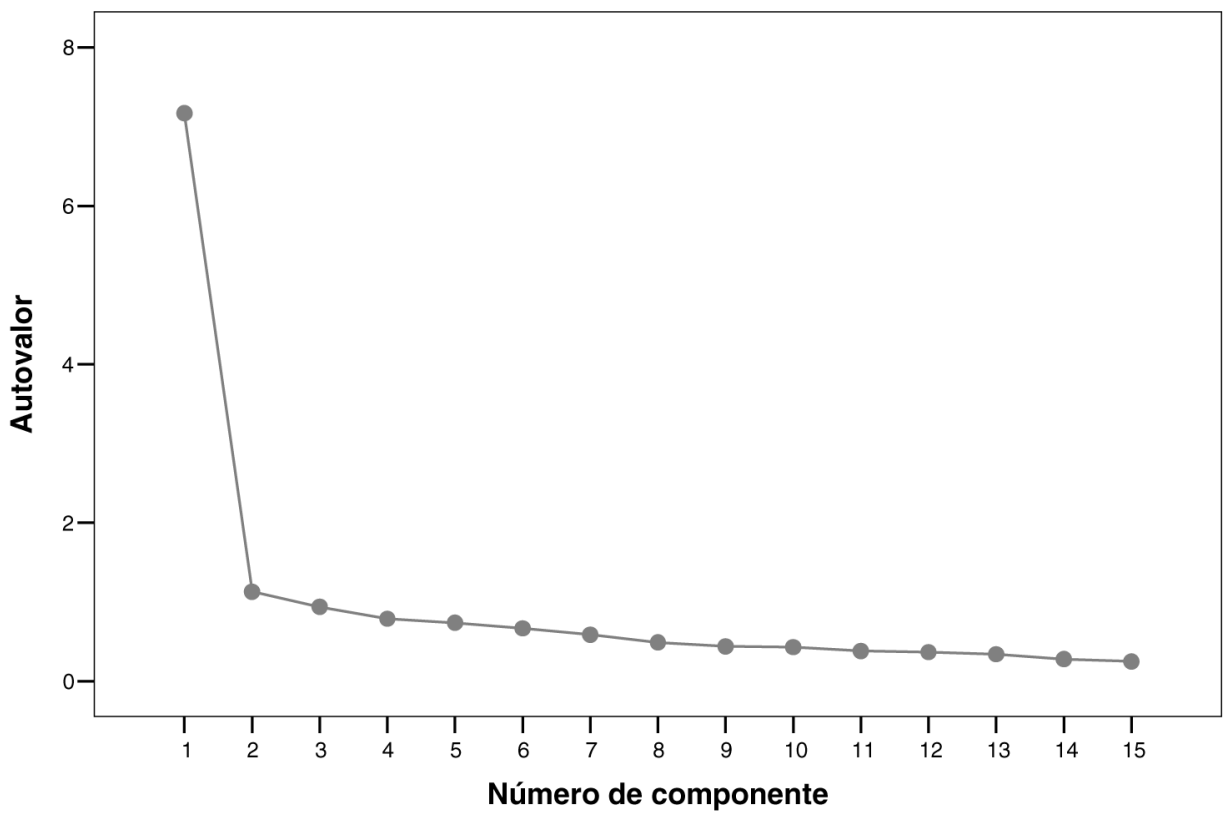

Tabla 2.Estructura factorial del Cuestionario de Acoso Psicológico Percibido

Solución bifactorial

Solución unifactorial

Factor 1

Factor 2

1. Percibo cierta inseguridad en mi puesto de trabajo por motivos imprecisos, difíciles de determinar.

2. Se producen discusiones entre compañeros de manera que se nota tensión en el ambiente.

3. Los superiores manifiestan abierta y públicamente su disconformidad con el trabajo realizado por los subordinados.

4. Algunas personas son capaces de mentir, levantar falsos testimonios con la finalidad de hacer daño a otros.

5. Algunos compañeros manifiestan abiertamente desprecio y burlas hacia otros.

6. Si no se hace lo que algunos imponen por detrás te hacen el vacío y no te dejan vivir.

7. Siento que no puedo confiar en los compañeros.

8. Los más fuertes suelen conseguir lo que se proponen a costa de otros.

9. Es difícil estar seguro de lo que haces, por algún lado te pueden atacar.

10. Determinadas personas pueden llegar a agredir verbalmente a los compañeros.

11. Cuando se lo proponen, algunos pueden hacerle la vida imposible a alguien.

12. Relacionarme con algunas personas me produce malestar, prefiero evitarlas.

13. Siento como si me estuvieran acosando en mi lugar de trabajo.

14. Algunas personas atribuyen y acusan a otros con sus propios defectos.

15. En alguna ocasión me he sentido amenazado en mi lugar de trabajo.

$\begin{array}{lll}.364 & .364 & .707 \\ .598 & .598 & -.019 \\ .577 & .577 & .434 \\ .785 & .785 & -.176 \\ .746 & .746 & -.212 \\ .787 & .787 & .099 \\ .663 & .663 & .125 \\ .696 & .696 & .098 \\ .726 & .726 & .134 \\ .760 & .760 & -.351 \\ .794 & .794 & -.262 \\ .667 & .667 & -.136 \\ .688 & .688 & .187 \\ .739 & .739 & -.227 \\ .656 & .656 & .123\end{array}$


Tabla 3: Evidencia de validez (correlación del CAPP con las medidas de salud del GHQ-28 y con las tres dimensiones del burnout del MBI)

\begin{tabular}{lcl}
\hline Variable & & r Spearman \\
\hline & Salud general & \\
Síntomas somáticos & & $.233^{*}$ \\
Ansiedad e insomnio & & $.331^{*}$ \\
Disfunción social & & $.222^{*}$ \\
Depresión & & $.295^{*}$ \\
GHQ-28 & Burnout & $.332^{*}$ \\
Cansancio emocional & & $.395^{*}$ \\
Despersonalización & & $.372^{*}$ \\
Realización personal & & $-.300^{*}$ \\
\hline
\end{tabular}

Nota. $* \mathrm{p}<.01$

zación personal, siendo las correlaciones con el resto de las escalas positivas y significativas (ver Tabla 3 ). Concretamente, las subescalas del GHQ-28 ansiedad e insomnio junto con depresión han sido las correlaciones positivas más altas con el CAPP. En todo caso, la correlación del CAPP con GHQ-28 (o distrés psicológico) es significativa, aunque moderada $(\mathrm{r}=.33)$. La correlación más alta del CAPP con las dimensiones del MBI la encontramos con cansancio emocional ( $\mathrm{r}=$ .39), siendo la más baja la correlación negativa con realización personal $(\mathrm{r}=-.30)$. En conjunto, tenemos una asociación significativa entre acoso psicológico subjetivo y los autoinformes de distrés psicológico mediante el Cuestionario de Salud General de Goldberg, con sus 4 subescalas (síntomas somáticos, ansiedad e insomnio, disfunción social y depresión), y las tres dimensiones del burnout según el MBI (cansancio emocional, despersonalización y realización personal).

\section{Discusión}

La relevancia que ha adquirido el concepto bullying o acoso en el trabajo en los últimos años, hace necesario llegar a un acuerdo, por un lado, tanto en las definición como en la prevalencia del fenómeno (Agerwold, 2007) para poder hacerle frente cuando se detecta y, sobre todo, para adelantarse al daño y sus consecuencias con una prevención efectiva en el ámbito del bienestar laboral. También se requiere acuerdo en la definición de acoso en el trabajo, particularmente para el uso del sistema de medida más apropiado de recogida de información (Cowie, Naylor, Rivers, Smith y Pereira, 2002), ya se trate del enfoque subjetivo (con técnicas de autoinforme) o del objetivo (por observación de conductas).

Como una contribución a la temática, hemos querido aportar un instrumento útil de evaluación del acoso psicológico percibido. Se precisan procedimientos y técnicas de recogida de información que, orientados a las variables cruciales del problema, resulten verdaderamente útiles para atajarlo y compensar satisfactoriamente el daño causado a las víctimas, o bien para actuar acertadamente en su prevención dentro del campo más amplio de la violencia ocupacional. Para Cantera, Cervantes y Blanch (2008), estamos ante una ya extensa información empírica sobre el problema de la violencia ocupacional, pero "heterogénea, fragmentaria y dispersa". El presente trabajo contribuye, según creemos, a enriquecer el enfoque subjetivo de la medición mediante autoinforme, aportando una escala fácilmente aplicable que posee las propiedades psicométricas necesarias. Con esta medida se evalúa, como querían Hoel et al. (1999), la percepción subjetiva que tienen las personas sobre el trato inapropiado que reciben en su lugar de trabajo.

El Cuestionario de Acoso Psicológico Percibido cuenta con adecuadas propiedades psicométricas de confiabilidad y validez. La escala presenta una buena consistencia interna, ya que excede del criterio mínimo recomendado de .70 por Nunnally y Bernstein (1994). La estructura unifactorial de la escala y la consistencia interna de la misma reflejan que todos los ítems son adecuados indicadores del constructo. Con respecto a la validez de constructo del Cuestionario de Acoso Psicológico, como se esperaba, se hallaron correlaciones significativas entre el acoso percibido y mayor burnout (más cansancio emocional, mayor despersonalización y menor realización personal). Los resultados son equivalentes a los de otros estudios previos que han relacionado el mobbing con el estrés ocupacional (ver Hoel, Zapf y Cooper, 2002; Mikkelsen y Einarsen, 2001) y más específicamente con el síndrome de burnout (Einarsen, Matthiesen y Skogstad, 1998). También esta asociación entre mobbing y burnout ha sido hallada por Moreno-Jiménez, Rodríguez-Muñoz, Martínez y Gálvez (2007) con mayores niveles de cansancio emocional, de pérdida de energía y de fatiga mental, así como más estrés percibido en las víctimas de acoso. Asimismo, se encontraron correlaciones significativas entre el acoso percibido y las diversas medidas de salud. La relación entre mobbing y salud ha sido investigada en numerosos estudios, como, por ejemplo, el de Hansen, Hogh, Perrson, Karlson, Garde y Ørbæk (2006), en el que se informa de que los empleados acosados padecen más síntomas somáticos, depresión, ansiedad y afecto negativo que los no acosados. También Moreno-Jiménez et al. (2006) y García-Izquierdo et al. (2006) han hallado que el acoso psicológico en el trabajo se asocia con dimensiones de deterioro de la salud psicológica del GHQ-28. Ya Leymann (1996) había remarcado que el mobbing tenía consecuencias tales como pérdida de salud, síntomas somáticos y enfermedades detectadas, así como también ansiedad y depresión. No obstante, debe tenerse en cuenta que el Cuestionario de Acoso Psicológico aquí presentado no es un cuestionario de diagnóstico 
clínico. Para discriminar si un trabajador es o no víctima de acoso se necesitan investigaciones con referentes clínicos, con el fin de contar con criterios diagnósticos especialmente útiles en los casos legales, tal como apuntara Leymann (1996).

Respecto a posibles perspectivas de futuro para este ámbito de estudio propuestas por Hoel y Cooper (2001) y Einarsen y Hauge (2006), una mejor medición del acoso contribuirá a una decisión más acertada sobre qué aspectos del fenómeno se pretenden investigar y, en consecuencia, a una mejor comprensión del proceso de acoso, de sus antecedentes y de la experiencia de las personas afectadas. Una vez que esté bien delimitado el campo del acoso en el lugar de trabajo (workplace bullying, harassment o mobbing), y su percepción subjetiva, ha de ampliarse el campo de estudio en diferentes direcciones en investigaciones futuras, como han sugerido los autores: los orígenes del acoso, psicológicos o de clima laboral (Einarsen, Raknes y Matthiesen, 1994; Vartia, 1996; Zapf, 1999; Muñoz, Guerra, Baron y Munduate, 2006), las consecuencias del acoso y el deterioro del bienestar en las víctimas y los observadores del mismo (Vartia, 2001; Einarsen y Hauge, 2006), la implementación de medios para su prevención (Salin, 2006, 2008), o los aspectos legales y éticos del acoso en el lugar de trabajo (LaVan y Martin, 2007; Bonafons, Jehel, Irigoyen y Coroller-Bequet, 2008).
Aunque los aspectos centrales del acoso psicológico en el lugar de trabajo no coinciden con los centrales en el síndrome de burnout (Dollard, Dormann, Boyd, Winefield y Winefield, 2003), no quiere decir que no exista entre ellos una relación digna de ser más ampliamente investigada. Ello se debe a que existen otras condiciones del trabajo en servicios humanos, aparte de las relaciones con personas en tanto que pacientes o clientes, que causan o pueden causar burnout. De especial importancia es la insatisfacción laboral de los profesionales de servicios humanos (Dollard et al., 2003; Einarsen et al, 1998; García-Izquierdo et al., 2006), insatisfacción que pone en peligro el bienestar psicológico de los empleados y su eficacia profesional. Si, por añadidura, se da también percepción subjetiva de acoso psicológico o riesgo del mismo en el lugar de trabajo, podemos estar ante un clima laboral con grupos de alto riesgo de mobbing y, en consecuencia, de mayores niveles de insatisfacción laboral y de burnout.

El ambiente psicosocial del trabajo y las particulares reacciones de los individuos al estrés (Agervold y Mikkelsen, 2004) son, sin duda, dos ámbitos de conocimiento que interesan sobre todo a quienes se proponen promover la salud psicológica en los lugares de trabajo, finalidad a la que resultará útil la información recogida mediante el CAPP.

\section{Extended Summary}

Mobbing is defined as a way of interpersonal aggression in work environment (Salin, 2003). Different terms have been used to describe this interpersonal aggression. Researchers indicate that sometimes the term bullying is used (e.g., Einarsen, Hoel, Zapf, and Cooper, 2003) while other times mobbing is preferred to mean the same phenomenon (LaVan and Martin, 2007). In the "Special issue on mobbing in the workplace", MorenoJiménez and Rodríguez-Muñoz (2006) used indistinctly the terms workplace mobbing (e.g., Einarsen and Hauge, 2006) and workplace bullying (e.g., MorenoJiménez, Rodríguez-Muñoz, Moreno, and Garrosa, 2006) whereas in the United States other terms are also used, such as emotional abuse (Keashly, 1998) and workplace aggression (Neuman and Baron, 1998). Among those who prefer to use workplace bullying, this expression is equivalent to harassment (v.g., Salin, 2008). Typically, bullying/harassment is an accumulation of a lot of minor events resulting in a systematic maltreatment pattern. In Europe, Hanz Leymann (1990, 1996) pioneered the study of psychological terror in the workplace, which he named mobbing. According to him, the concept defines the link between hostile words and threats expressed by one or several workers towards a third person (the victim) placing him/her in a situation of disadvantage, with a high risk of being dismissed from his job. This situation persists a long period of time (Leymann, 1996). To be termed mobbing, an event has to meet three requirements: 1) events must be felt as hostile, 2) events must occur at least once a week, and 3 ) they must persist over a period of time -six months at least. According to Einarsen et al. (2003), mobbing in the workplace involves irritating, offending, excluding socially or hindering someone from doing his/her work. Different events like being assigned low demanding tasks (below one's own capacity), being the target of coworkers jokes, or being excluded from social get-togethers can take place daily in a workplace. Mobbing behaviors are aimed at humiliating, intimidating, terrifying, or punishing someone -the victim.

There are two main approaches to measuring mobbing (Einarsen et al., 2003): (1) the subjective method, which evaluates victim's self perception; and (2) the objective measure or objective exposure to mobbing events. Both approaches are subject to criticism (see Hoel, Rayner, and Cooper, 1999; Mikkelsen and Einarsen, 2001; Salin, 2006). The frequency of mobbing ranges from 8 to 15 percent when measured by a subjective method and from 2 to 10 percent when it is measured by an objective method (Zapf et al., 2003). There are a number of reasons to question the validity of the objective method. First, the cut-off score to decide 
whether or not someone is suffering mobbing is chosen in an arbitrary manner. Second, each technique uses a different number of events (from 26 in the NAQ to 45 in the LIPT). In addition to that, not all the events have the same level of severity. Hence, sometimes even a low exposure to negative events can be considered mobbing (Notelaers et al., 2006).

We have chosen the Perceived Mobbing Questionnaire $(C A P P)$ as a measure of mobbing. Contrary to other inventories that use an only item, CAPP is a subjective method that includes 15 items. There is only one item asking directly the person whether he or she believes he or she is suffering mobbing. The other items ask about feeling insecure and threatened, perception of aggression, feeling not to be in good terms with coworkers, perceiving that the work environment is intimidating, feeling frightened, and the tendency to be mobbed. The 15-item Perceived Mobbing Questionnaire is a five point Likert scale, ranging from "I don't feel it at all" to "I always feel it". The aim of this paper is to analyze the reliability of CAPP, its factor structure, and its correlation with burnout and health in a sample of Spanish workers.

\section{Method}

Participants were 390 workers (35 percent males) aged between 26 and 65 years (mean age of 38). The average time working in the company was 13 years -with a range of 1 to 40 . They completed three questionnaires: 1) Perceived Mobbing Questionnaire (CAPP); 2) the social workers version of the Maslach Burnout Inventory (MBI-HSS) (Maslach and Jackson, 1986) (reliability indices in this study were $\alpha=.79$ for personal accomplishment, $\alpha=.83$ for emotional exhaustion, and $\alpha=.54$ for depersonalization (getting the highest score in emotional exhaustion and depersonalization and the lowest in personal accomplishment is an indication of burnout); 3) the General Health Questionnaire (GHQ-28 by Goldberg and Williams, 1996) is a measure of psychological distress with four scales: somatic symptoms, anxiety and insomnia, social dysfunction and depression (reliability in this study was $\alpha=.82$ for somatic symptoms, $\alpha=.90$ for anxiety and insomnia, $\alpha=.76$ for social dysfunction and depression, and $\alpha=.92$ for the total scale).

\section{Results}

Descriptive analyses were performed for all variables in the CAPP, along with reliability analysis and exploratory factor analysis -using the principal component method and orthogonal rotation. Since data did not show normal distribution, Spearman's correlation coefficients were calculated between variables. The Kolmogorov-Smirnov's test demonstrated lack of nor- mality in the distribution of variables. According to measures of central trend and the scales score range, scores were far from the maximum (75 points) for mobbing. The same applies to GHQ subscales -somatic symptoms, anxiety-insomnia, social dysfunction, and depression are far from the maximum score (21 points); likewise, general health score as indicated by psychological distress moves away from the maximum score $(84$ points). As for the scale of burnout, the average scores of emotional exhaustion and depersonalization move away from the maximum score (54 and 30 respectively); on the contrary, the average score (38) in the area of personal accomplishment approaches the maximum score of 48 .

Exploratory factor analysis on CAPP results showed a KMO value of .937 that seems acceptable. The sphericity test was significant $\left(\chi^{2}=2883.86 ; \mathrm{df}=105\right.$; $\mathrm{p}=.001)$ implying that factor analysis on these items was suitable. Regarding the number of factors, rule K1 suggests two factors, although scree plot suggests one factor that explains $47.78 \%$ of the variance and a second factor explaining $7.52 \%$ of the variance. Thus, the one-factor structure is the best solution. Cronbach's alpha of the scale was .92 .

In order to calculate validity, the scale was compared with three subscales of MBI and GHQ-28 subscales. Results showed that CAPP correlated negatively with personal accomplishment, whereas correlations with the other scales were positive -all correlations were significant.

\section{Discussion}

An agreement on definitions of mobbing is needed, in order to determine its prevalence (Agerwold, 2007). Only in this manner a contribution will be possible to detect mobbing and to prevent it and its consequences in the workplace. Such an agreement will also allow us to achieve a more appropriate method to collect information (Cowie, Naylor, Rivers, Smith, and Pereira, 2002), whether subjectively or objectively.

We suggest a useful instrument to assess mobbing, a self-report technique -the Perceived Mobbing Questionnaire. CAPP is an easy-to-administer questionnaire with sound psychometric properties. Hoel et al. (1999) recommended CAPP as an adequate subjective measure of perceived harassment in the workplace. No doubt, the psycho-social environment in the workplace and personal reactions to stress (Agervold and Mikkelsen, 2004) are subjects of interest for people involved in the promotion of psychological health in the workplace. CAPP may help achieve this goal.

\section{Referencias}

Agerwold, M. (2007). Bullying at work: A discussion of definitions and prevalence, based on an empirical study. 
Scandinavian Journal of Psychology, 48, 161-172. environment and individual stress reactions. Work and Stress, 18 (4), 336-351.

Bonafons, C., Jehel, L., Irigoyen, M. F. y Coroller-Bequet, A. (2008). Clarifying the definition of bullying. L'Encéphale. Revue de Psychiatrie Clinique, Biologique et Thérapeutique, 34, 419-426.

Bowling, N. A. y Beehr, T. A. (2006). Workplace harassment from the victim's perspective: A theoretical model and meta-analysis. Journal of Applied Psychology, 91 (5), 998-1012.

Cantera, L. M., Cervantes, G. y Blanch, J. M. (2008). Violencia ocupacional: El caso de los profesionales sanitarios. Papeles del Psicólogo, 29, 49-58.

Cowie, H., Naylor, P., Rivers, I., Smith, P. K. y Pereira, B. (2002). Measuring workplace bullying. Aggression and Violent Bahavior, 7, 33-51.

Dollard, M. F., Dormann, C., Boyd, C. M., Winefield, H. R. y Winefield, A. H. (2003). Unique aspects of stress in human service work. Australian Psychologist, 38 (2), 8491.

Einarsen, S. y Hauge, L. J. (2006). Antecedentes y consecuencias del acoso psicológico en el trabajo: una revisión de la literatura. Revista de Psicología del Trabajo y de las Organizaciones, 22 (3), 251-273.

Einarsen, S. y Hoel, H. (2001). The validity and development of the Revised Negative Acts Questionnaire. Paper presented at the European Congress of Work and Organizational Psychology, Praga.

Einarsen, S., Hoel, H., Zapf, D. y Cooper, C. L. (2003). The concept of bullying at work. The European tradition. En S. Einarsen, H. Hoel, D. Zapf, y C. L. Cooper (Eds.), Bullying and emotional abuse in the workplace. International perspectives in research and practice (pp. 3-30). Londres: Taylor \& Francis.

Einarsen, S., Matthiesen, S.B. y Skogstad, A. (1998). Bullying, burnout and well-being among assistant nurses. Journal of Occupational Health and Safety - Australia and New Zealand, 14, 263-268.

Einarsen, S., Raknes, B. I. y Matthiesen, S. B. (1994). Bullying and harassment at work and their relationship to work environment quality. An exploratory study. European Journal of Work and Organizational Psychology, 4, 4, 381-401.

García-Izquierdo, M., Llor, B., García-Izquierdo, A. L. y Ruiz, J. A. (2006). Bienestar psicológico y mobbing en una muestra de profesionales de los sectores educativo y sanitario. Revista de Psicología del Trabajo y de las Organizaciones, 22 (3), 381-395.

Goldberg, D. y Williams, P. (1996). Cuestionario de Salud General GHQ. Barcelona: Masson.

González de Rivera, J. L. y Rodríguez, M. (2003). Cuestionario de estrategias de acoso psicológico: el LIPT60 (Leymann Inventory of Psychological Terrorization) en versión española. Psiquis, 24 (2), 17-27.

Hansen, Ä. M., Hogh, A., Persson, R., Karlson, B., Garde, A. H. y Ørbæk, P. (2006). Bullying at work, health outcomes, and physiological stress response. Journal of
Psychosomatic Research, 60, 63-72.

Hoel, H. y Cooper, C. L. (2001). Origins of bullying: Theoretical frameworks for explaining workplace bullying. En N. Tehrani (Ed.), Building a culture of respect: Managing bullying at work (pp. 3-19). Londres: Taylor \& Francis.

Hoel, H., Rayner, C. y Cooper, C. L. (1999). Workplace bullying. En C. L. Cooper, y I. I. Robertson (Eds.), International Review of Industrial and Organisational Psychology (pp. 195-230). Chichester: John Wiley \& Sons.

Hoel, H., Zapf, D. y Cooper, C. L. (2002). Workplace bullying and stress. Historical and Current Perspectives on Stress and Health, 2, 293-333.

Keashly, L. (1998). Emotional abuse in the workplace: Conceptual and empirical issues. Journal of Emotional Abuse, 1, 85-117.

Kerlinger, F. N. (2001). Investigación del comportamiento: métodos de investigación en ciencias sociales. México: Mc Graw Hill.

LaVan, H. y Martin, Wm. M. (2008). Bullying in the U.S. workplace: Normative and process-oriented ethical approaches. Journal of Business Ethics, 83, 147-165.

Leymann, H. (1990). Manual of the LIPT questionnaire for assessing the risk of psychological violence at work. Estocolmo: Violen.

Leymann, H. (1996). The content and development of mobbing at work. European Journal of Work and Organisational Psychology, 5 (2), 165-184.

Maslach, C. y Jackson, S. E. (1986). Maslach Burnout Inventory manual ( $2^{\text {nd }}$ ed.). Palo Alto, CA: Consulting Psychologists.

Matthiesen, S. B., Raknes, B. I. y Røkkum, O. (1989). Mobbing pa arbeidsplassen [Bullying at the workplace]. Tidsskrift for Norsk Psykologforening, 26, 761-774.

Mikkelsen, E. y Einarsen, S. (2002). Relationships between exposure to bullying at work and psychological and psychosomatic health complaints: The role of state negative affectivity and generalized self-efficacy. Scandinavian Journal of Psychology, 43, 397-405.

Mikkelsen, E., y Einarsen, S. (2001). Bullying in Danish work-life: Prevalence and health correlates. European Journal of Work and Organizational Psychology, 10, 393413.

Morán, C. (2007). Acoso psicológico y burnout en profesionales de recursos humanos. En J. Romay (Ed.), Perspectivas y retrospectivas de la Psicología Social en los albores del siglo XXI (pp. 249-256). Madrid: Biblioteca Nueva.

Moreno-Jiménez, B. y Rodríguez-Muñoz, A. (2006). Introducción. Número monográfico sobre acoso psicológico en el trabajo: una perspectiva general. Revista de Psicología del Trabajo y de las Organizaciones, 22 (3), 245-249.

Moreno-Jiménez, B., Rodríguez-Muñoz, A., Garrosa, E. y Morante, M. E. (2005). Antecedentes organizacionales del acoso psicológico en el trabajo: un estudio exploratorio. Psicothema, 17 (4), 627-632. 
Moreno-Jiménez, B., Rodríguez-Muñoz, A., Martínez, y Gálvez, M. (2007). Assessing workplace bullying: Spanish validation of a reduced version of the Negative Acts Questionnaire. The Spanish Journal of Psychology, 10 (2), 449-457.

Moreno-Jiménez, B., Rodríguez-Muñoz, A., Moreno, Y., y Garrosa, E. (2006). El papel moderador de la asertividad y la ansiedad social en el acoso psicológico en el trabajo: dos estudios empíricos. Revista de Psicología del Trabajo y de las Organizaciones, 22 (3), 363-380.

Muñoz, H., Guerra, J. M., Baron, M. y Munduate, L. (2006). El acoso psicológico desde una perspectiva organizacional. Papel del clima organizacional y los procesos de cambio. Revista de Psicología del Trabajo y de las Organizaciones, 22 (3), 347-362.

Neuman, J. H. y Baron, R. A. (1998). Workplace violence and workplace aggression: Evidence concerning specific forms, potential causes, and preferred targets. Journal of Management, 24, 391-419.

Notelaers, G., Einarsen, S., De Witte, H. y Vermunt, J. K. (2006). Measuring exposure to bullying at work: The validity and advantages of the latent class cluster approach. Work \& Stress, 20 (4), 288-301.

Nunnally, J. C. y Bernstein, I. H. (1994). Psychometric theory ( $3^{\text {rd }}$ ed.). Nueva York: McGraw-Hill.

Salin, D. (2003). Ways of explaining workplace bullying: A review of enabling, motivating and precipitating structures and processes in the work environment. Human Relations, 56 (10), 1213-1232.

Salin, D. (2006). ¿Se preocupan las organizaciones por el acoso psicológico en el trabajo?: Percepciones de los trabajadores sobre las medidas organizacionales contra el acoso psicológico. Revista de Psicología del Trabajo y de las Organizaciones, 22 (3), 413-432.

Salin, D. (2008). The prevention of workplace bullying as a question of human resource management: measures adopted and underlying organizational factors. Scandinavian Journal of Management, 24, 221-231.

Unal, A. y Karaadhmet, E. (2007). Mobbing among employees in Bursa, Turkey: A cross-sectional survey study. Neurology Psychiatry and Brain Research, 14 (4), 159-154.

Vartia, M. (1996). The sources of bullying: Psychological work environment and organizational climate. European Journal of Work and Organizational Psychology, 5 (2), 203-214.

Vartia, M. (2001). Consequences of workplace bullying with respect to the well-being of its targets and the observers of bullying. Scandinavian Journal of Work, Environment and Health, 27, 63-69.

Zapf, D. (1999). Organisational, work group related and personal causes of mobbing/bulling at work. International Journal of Manpower, vol. 20, $\mathrm{n}^{\circ} 1 / 2,70-85$.

Zapf, D. y Einarsen, S. (2003). Individual antecedents of bullying: Victims and perpetrators. En S. Einarsen, H. Hoel, D. Zapf y C. L. Cooper (Eds.), Bullying and emotional abuse in the workplace. International perspectives in research and practice (pp. 165-184). Londres: Taylor $\&$ Francis.

Zapf, D., Einarsen, S., Hoel, H. y Vartia, M. (2003). Empirical findings on bullying in the workplace. En S. Einarsen, H. Hoel, D. Zapf, y C. L. Cooper (Eds.), Bullying and emotional abuse in the workplace. International perspectives in research and practice (pp. 103-126). Londres: Taylor \& Francis.

Manuscrito Recibido: 25/5/2008

Revisión Recibida: 20/3/2009

Aceptado: 26/3/2009 